\title{
Genomic Analysis, Sequence Diversity, and Occurrence of Apple necrotic mosaic virus, a Novel llarvirus Associated with Mosaic Disease of Apple Trees in China
}

Fei Xing, College of Horticulture, China Agricultural University, Beijing 100193; and Institute of Plant Protection, Chinese Academy of Agricultural Sciences, Beijing 100193; Berhanu Lemma Robe and Zhixiang Zhang, Institute of Plant Protection, Chinese Academy of Agricultural Sciences, Beijing 100193; Hongqing Wang, ${ }^{\dagger}$ College of Horticulture, China Agricultural University, Beijing 100193; and Shifang Li, ${ }^{\dagger}$ Institute of Plant Protection, Chinese Academy of Agricultural Sciences, Beijing 100193

\begin{abstract}
China accounts for over $50 \%$ of apple production worldwide. Very recently, a novel ilarvirus, Apple necrotic mosaic virus (ApNMV), was isolated from apple trees showing mosaic symptoms in Japan. This study compared different types of mosaic symptoms observed in apple trees in China under field conditions. Complete nucleotide sequences were obtained for six isolates of ApNMV. The genomic components varied in size from 3,378 to $3,380 \mathrm{nt}$ (RNA1), 2,778 to 2,786 nt (RNA2), and 1,909 to 1,955 nt (RNA3), respectively. Although nucleotide sequence similarities with subgroup 3 ilarviruses were low (49.2 to $64.3 \%$ ), results of phylogenetic analysis indicated that Chinese

ApNMV isolates were clustered in subgroup 3 together with Prunus necrotic ring spot virus (PNRSV) and Apple mosaic virus (ApMV). Apple mosaic disease occurred widely in apple producing areas of China with a very high percentage $(92.1 \%, 268$ out of 291) of symptomatic trees being infected with ApNMV but not with ApMV. The data suggested that ApNMV might be the main pathogen causing apple mosaic disease in China. The genomes of the six studied Chinese ApNMV isolates demonstrated substantial sequence diversity. Here, we demonstrated a strong association of ApNMV with the mosaic disease of apple trees in China.
\end{abstract}

Apple (Malus domestica) is one of the most economically important fruit crops grown worldwide. China accounts for over 2.4 million ha of apple grown with a fruit production of 40 million tons, which accounts for half of the world production. Apple trees are perennial woody plants susceptible to diseases including viruses or viroids. $A p$ ple stem pitting virus (ASPV), Apple stem grooving virus (ASGV), Apple chlorotic leaf spot virus (ACLSV), and Apple scar skin viroid (ASSVd) are the main viruses or viroid infecting apple trees in China (Hu et al. 2015; Ji et al. 2013).

Apple mosaic disease (outside of China typically associated with infection by the ilarvirus, Apple mosaic virus [ApMV]) also occurs widely, and has been a severe threat to the development of the apple industry with high numbers of symptomatic trees being recorded in some apple orchards in China ( $\mathrm{Li}$ et al. 2002). Apple mosaic disease was first reported in Europe and was one of the viral diseases that could be transmissible by budding and grafting (Bradford and Joley 1933; Fukushi and Tahama 1960; Fulton 1972; Petrzik and Lenz 2011). Apple leaves with apple mosaic disease show pale yellow to bright irregular spots or bands along leaf veins or mosaic pattern and chlorosis (Grimová et al. 2016; Petrzik and Lenz 2011). ApMV was believed to be the only causal agent of apple mosaic disease for a long period of time. However, for nearly a decade, ApMV was not detected in our surveys of apple mosaic disease in China even though symptoms similar to those described for ApMV were regularly observed in apple trees (Liang et al. 2016; and unpublished observations).

Very recently, a novel virus, Apple necrotic mosaic virus (ApNMV), was identified in apple trees with mosaic disease in Japan

${ }^{\dagger}$ Corresponding authors: H. Q. Wang, E-mail: wanghq@ cau.edu.cn; and S. F. Li, E-mail: sfli@ippcaas.cn

Funding: This work was supported by key inter-governmental projects of the national key research and development program (2017YFE0110900) and the grant from the Special Fund for Agro-scientific Research in the Public Interest (201203076).

*The $\boldsymbol{e}$-Xtra logo stands for "electronic extra" and indicates that two supplementary tables and three supplementary figures are published online.

Accepted for publication 13 March 2018.

(c) 2018 The American Phytopathological Society that originated from China (Noda et al. 2017). This new virus was associated with mosaic symptoms similar to those induced by ApMV (Noda et al. 2017). ApNMV contains three single-stranded positive-sense RNA segments and belongs to the genus Ilarvirus of the family Bromoviridae (Noda et al. 2017). Virus replication depends on two nonstructural proteins, methyltransferase/NTP-binding helicase (MET/HEL) and RNA polymerase (POL), encoded by monocistronic RNA1 and RNA2 (Noda et al. 2017). RNA3 encodes a nonstructural movement protein (MP) at $5^{\prime}$ terminal, and a coat protein (CP) at $3^{\prime}$ terminal (Noda et al. 2017).

The objective of this study was to characterize the genetic diversity of ApNMV in China and gain a clear idea of the association between ApNMV and apple mosaic disease of apple trees in China. This information is needed for identification of the causal agent of mosaic disease in apple plants in China. In addition, the whole genome sequences of six ApNMV isolates from China were determined and analyzed. To the best of our knowledge, this is the first analysis of the ApNMV genetic diversity from apple trees in China, establishing a strong association between ApNMV presence in apple trees and apple mosaic disease symptoms in China.

\section{Materials and Methods}

Plant materials and virus isolates. Apple leaves showing mosaic symptoms were collected from the major apple-growing provinces and regions (Shandong, Shaanxi, Shanxi, Gansu, Xinjiang, and Beijing) in China during 2013 to 2017 from a total of 291 samples (Table 1). Leaf samples randomly collected from different parts of the same trees were used for viral disease diagnosis. The collected samples were stored at $-80^{\circ} \mathrm{C}$ until total RNAs were extracted. Details of the complete nucleotide sequences of six Chinese ApNMV isolates, including their place of origin, year of collection, apple cultivar, symptoms, and accession number are shown in Table 2.

RNA extraction and complete genome sequencing. Total RNAs were extracted from six apple leaf samples showing mosaic symptoms (AM27, AM36, AM72, AM75, AM95, and AM125) using an RNAprep Pure Plant Kit (TianGen, Beijing, China) following the manufacturer's instructions. E. coli polymerase (NEB, Beijing, China) was used to add $3^{\prime}$ poly A tails to viral RNAs. M-MLV reverse transcription was used for the synthesis of first-strand cDNAs with a random hexamer primer. ApNMV genomic segments were amplified by PCR using Phusion High-Fidelity DNA Polymerase (Thermo Scientific, U.S.A.). The RNA3 partial genome of AM75 was amplified 
using the forward degenerate primer R3S1 designed from the conserved domains of ApMV and PNRSV and the reverse primer ApNMV-R. Amplification of $5^{\prime}$ and $3^{\prime}$ cDNA ends were carried out using the SMARTer RACE $5^{\prime} / 3^{\prime}$ Kit (Clontech, U.S.A.) following the manufacturer's instructions using the primer sets of 5R3GSP1/2 and ApNMV-F, respectively. The primer pairs of R1S1/R1A1 and R2S1/ApnA1, R2S2/R2A2, or ApnS1/R2A2 were used for the amplification of RNA1 and RNA2 genomic segments. The primer pairs of 5R1GSP1/2, 5R2GSP1/2, and 3R1GSP1, 3R2GSP1 were used for the amplification of $5^{\prime}$ and $3^{\prime}$ cDNA ends of RNA1 and RNA2, respectively (Supplementary Fig. S1). Finally, all consensus sequences of the three RNA segments were aligned and assembled to obtain the complete ApNMV genome sequence from AM75. Subsequently, the complete genomes of ApNMV from other five apple leaf samples (AM27, AM36, AM72, AM95, and AM125) were obtained (Supplementary Fig. S1). All the primers used for sequencing of ApNMV are listed in Supplementary Table S1.

Phylogenetic analysis. Nucleotide and deduced amino acid sequences from the four ORFs were aligned with the homologous sequences of other ilarviruses using ClustalX 1.83. The phylogenetic relationships of the four aligned ORF sequences were assessed by MEGA version 6 (Tamura et al. 2013) using the neighbor-joining method (Saitou and Nei 1987) with 1,000 bootstrap replications.

RT-PCR detection of ApNMV and ApMV from apple mosaic diseased samples in China. For RT-PCR, the total RNAs were extracted from apple samples using CTAB extraction buffer (Lin et al. 2011; Zhang et al. 2012) and RNAprep Pure Plant Kit (TianGen, Beijing, China), and treated with DNase I at room temperature for $15 \mathrm{~min}$. The first-strand cDNAs were synthesized by reversetranscription reactions with random hexamer primers. Then the PCR was performed using the special primer set ApNMV-F/R and nested PCR using ApN-F2/R2 for the detection of ApNMV, and for ApMV detection, three primer sets ApMV-F1/R1, ApMV-F2/R2, and $\mathrm{CP}+2 / \mathrm{CP}-2$ located in the $\mathrm{CP}$ gene were used (Supplementary Table S2). The mastermix of samples was denatured at $94^{\circ} \mathrm{C}$ for $4 \mathrm{~min}$ and followed by 35 cycles of PCR ( 15 cycles of nested PCR) amplification with Taq polymerase (Bingda, Beijing, China) at $94^{\circ} \mathrm{C}$ for $30 \mathrm{~s}$, $54^{\circ} \mathrm{C}$ for $30 \mathrm{~s}$, and $72^{\circ} \mathrm{C}$ for $50 \mathrm{~s}$, and a final step of $10 \mathrm{~min}$ at $72^{\circ} \mathrm{C}$.

\section{Results}

Apple mosaic symptoms in China. From 2013 to 2017, a survey was conducted, and a total of 291 apple mosaic leaves were collected

Table 1. Geographical distribution and incidence of Apple necrotic mosaic virus (ApNMV) from apple mosaic trees in the major apple-producing areas in China

\begin{tabular}{llccc}
\hline Location & Cultivar & No. of mosaic samples & ApNMV & ApMV \\
\hline Shandong & Fuji & 166 & 164 & 0 \\
Shaanxi & Fuji & 74 & 59 & 0 \\
& Gala & 13 & 13 & 0 \\
Shanxi & Fuji & 20 & 17 & 0 \\
Gansu & Fuji & 3 & 1 & 0 \\
& Starking & 1 & 1 & 0 \\
Beijing & Fuji & 7 & 7 & 0 \\
Xinjiang & Unknown & 7 & 6 & 0 \\
Total & & 291 & 268 & 0 \\
Incidence rate & & - & $92.1 \%$ & $0.0 \%$ \\
\hline
\end{tabular}

in China (Table 1). The mosaic symptoms in apple trees varied from mild, moderate, to severe chlorosis, pale yellow mosaic to bright cream-colored irregular spots, rings. Bands and/or line patterns along the main veins were observed with uneven distribution on apple leaves (Fig. 1). Sometimes, brownish necrotic spots were also observed (Fig. 1C and M).

Complete nucleotide sequence analysis of the six Chinese ApNMV isolates. The complete nucleotide sequences of the six ApNMV isolates ranged in size from 3,378 to 3,380 nt (RNA1), 2,778 to $2,786 \mathrm{nt}$ (RNA2), and 1,909 to $1,955 \mathrm{nt}$ (RNA3), respectively, as shown in Table 3 . The sequence identity among the complete nucleotide sequences for six ApNMV isolates of RNAs 1 to 3 genomic segments was $97.7,95.0$, and $93.1 \%$, respectively. RNA1 encoded the MET/HEL protein (1,056 amino acids [aa]). RNA2 encoded the RNA POL protein of 855 to 867 aa, which contained the highly conserved motif sequence of glycine-aspartic acidaspartic acid (GDD) among ilarviruses at position 627 to 630 (or 626 to 629 ). Similarly, RNA3 encoded the MP (280 to 281 aa) and the CP protein (219 aa); the latter was predicted to be expressed from the subgenomic RNA4 (Pallas et al. 2013). The intergenic region (IR) between MP and CP genes plays a crucial role supposedly functioning as the promoter to initiate the transcription of RNA4 on RNA3 by the viral replicase (Hammond and Crosslin 1995). In our study, the IR was found to be 98 to 103 nt with GC contents of 43.2 to $47.6 \%$, which was different in length from that (145 nt) described in Japanese ApNMV isolate P129 (accession no. LC108995). The IR of ApNMV was U-rich (38.8 to $40.8 \%$ ). The $5^{\prime}$ untranslated regions (UTR) of RNA1 and RNA2 were 50 to 51 and 52 to $58 \mathrm{nt}$, respectively, and that of RNA3 showed a wide variation with 144 to $188 \mathrm{nt}$ in length. The 3' UTR of RNA1, RNA2, and RNA3 were 157 to $158 \mathrm{nt}$, 125 to $160 \mathrm{nt}$, and 160 to $161 \mathrm{nt}$ in length, respectively. Even though there was variability in length, the $3^{\prime}$ UTR of the complete nucleotide sequences of the six Chinese ApNMV isolates shared 91.9 to $99.2 \%$ nucleotide identities over the $3^{\prime}$-most $125 \mathrm{nt}$. The 5' UTR of RNA1 and RNA2 of six Chinese ApNMV isolates shared 95.1 and $84.8 \%$ nucleotide identities, respectively, while only $74.1 \%$ in RNA3.

Similar to the other ilarviruses (Hammond and Crosslin 1995; Herranz and Pallas 2004; James et al. 2010; Pallas et al. 2012, 2013; Sanchez-Navarro and Pallas 1994), the MP was predicted to have an RNA-binding domain (RBD) at aa position 55 to 87 and a hydrophobic region at aa position 88 to 109 , and the CP to have an $\mathrm{RBD}$ at aa position 25 to 50 and a zinc finger motif. A stem-loop structure assumed to bind onto the $\mathrm{CP}$ region to initiate the replication was predicted and the two AUGC motifs were present at the base of stems in the $3^{\prime}$ UTR (Fig. 2).

The similarities between Chinese ApNMV isolates of RNAs 1 to 3 genome sequences with the Japanese isolates (accession nos. LC108993; LC108994; LC108995) were 95.4 to $96.5 \%$ (RNA1), 88.5 to $94.5 \%$ (RNA2), and 86.2 to $91.1 \%$ (RNA3), respectively. The identities of MET/HEL, POL, MP, and CP with the Japanese ApNMV isolates were 95.4 to $96.6 \%, 88.6$ to $93.6 \%, 89.4$ to $93.9 \%$, and 90.6 to $94.1 \%$ based on the nucleotide sequences, and were 97.7 to $98.7 \%, 90.3$ to $93.6 \%, 93.6$ to $95.4 \%$, and 94.5 to $95.9 \%$ based on the amino acid sequences, respectively. The MET/HEL was highly conserved while the POL showed great variation among the four proteins.

Table 2. The Chinese Apple necrotic mosaic virus (ApNMV) isolates used in this study

\begin{tabular}{|c|c|c|c|c|c|}
\hline Isolate & Cultivar & Symptoms & Origin & Collection date & Accession numbers ${ }^{a}$ \\
\hline$\overline{\mathrm{AM}} 27$ & Gala & Mosaic & Yan'an, Shaanxi & July 2013 & KY808376-KY808378 \\
\hline AM36 & Gala & Mosaic & Yan'an, Shaanxi & July 2013 & KY808379-KY808381 \\
\hline AM72 & Fuji & Mosaic & Taian, Shangdong & April 2014 & KY808382-KY808384 \\
\hline AM75 & Fuji & Mosaic & Taian, Shangdong & April 2014 & KY808385-KY808387 \\
\hline AM95 & Fuji & Mosaic & Yan'an, Shaanxi & July 2013 & KY808388-KY808390 \\
\hline AM125 & Fuji & Mosaic & Yan'an, Shaanxi & July 2013 & KY808391-KY808393 \\
\hline
\end{tabular}

${ }^{a}$ RNA1, RNA2, and RNA3, respectively. 
Phylogenetic analysis of ApNMV and other ilarviruses. To understand the taxonomic position of this novel virus, phylogenetic analysis of the six Chinese ApNMV isolates (AM27, 36, 72, 75, 95, and 125), as well as one isolate (P129) originating from Japan, were constructed using the neighbor-joining method based on the amino acid sequences. The analysis clearly revealed that ApNMV was clustered in subgroup 3, which was almost consistent with the phylogenetic relationship constructed based on the nucleotide sequences (Fig. 3). Sequence analysis indicated that MET/HEL, POL, and CP genes of ApNMV were more closely related to PNRSV while it seemed that the MP gene was more closely related to Lilac leaf chlorosis virus (LLCV). The MET/HEL, POL, and CP of the seven ApNMV isolates were 68.5 to $69.0 \%, 60.3$ to $61.6 \%$, and 54.3 to $55.7 \%$ identical at the amino acid level and 64.5 to $65.0 \%, 57.9$ to $59.1 \%$, and 60.0 to $60.8 \%$ identical at the nucleotide level to those of PNRSV, respectively. The MPs were 56.5 to $58.3 \%$ identical at the amino acid level and 58.9 to $59.8 \%$ identical at the nucleotide level to those of LLCV, respectively.
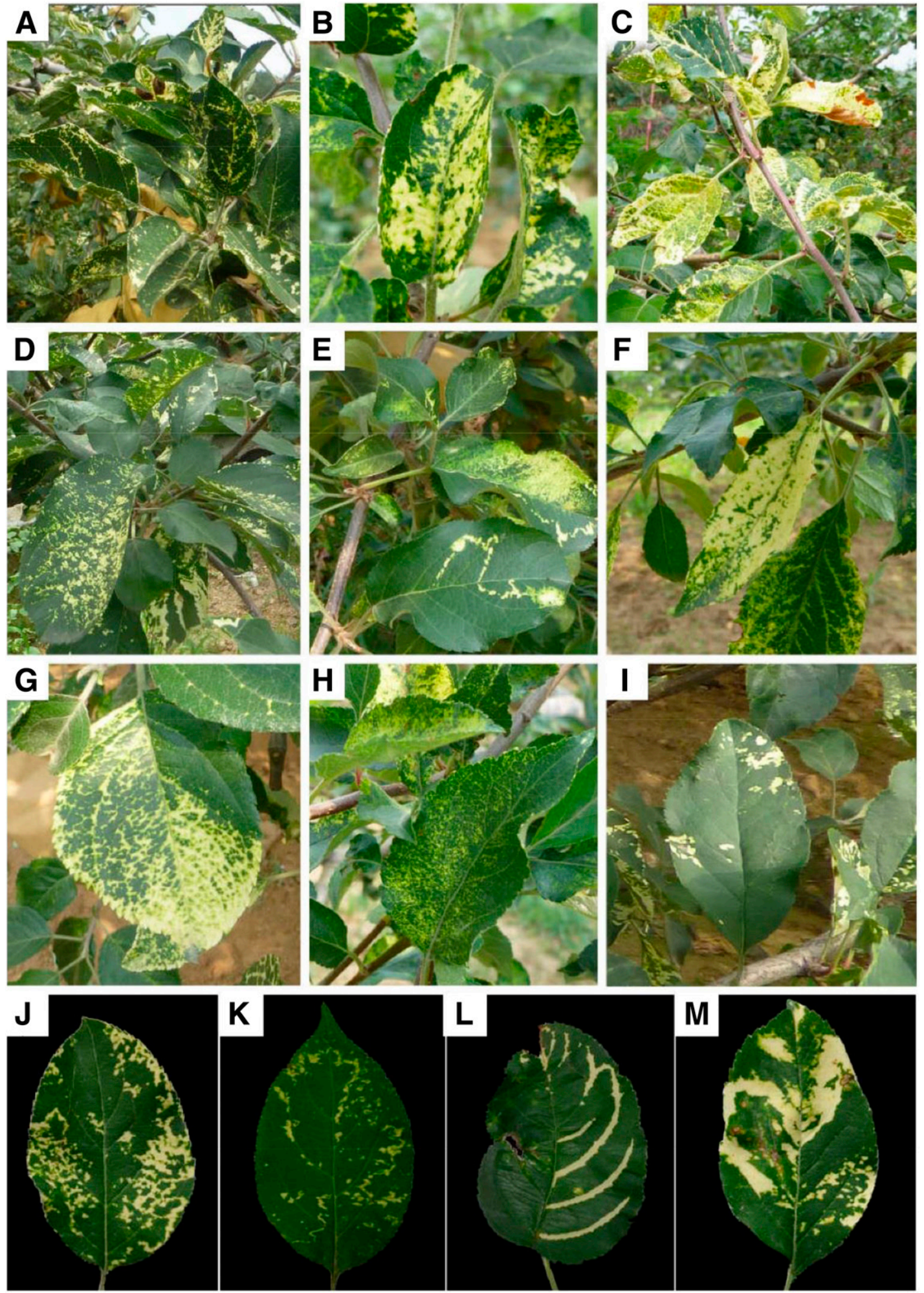

Fig. 1. Symptoms of Apple necrotic mosaic virus (ApNMV)-positive apple leaves in China. Chlorosis, pale yellow mosaic to bright cream-colored irregular spots, rings, bands, and/ or line patterns along the main veins were observed on apple leaves with uneven distribution (A to $\mathbf{M})$. Brownish necrotic spots were also observed (C, L, M). 
Relationship of ApNMV with ApMV and PNRSV based on sequence comparison. Since little information is available on the homology between ApNMV, ApMV, and PNRSV, sequence comparison was performed. The results showed that the RNA1 to 3 segments of the complete nucleotide sequences of six Chinese ApNMV isolates were 58.8 to $59.8 \%, 57.5$ to $58.3 \%$, and 49.2 to $50.6 \%$ identical to ApMV (accession nos. NC003464, NC003465, and NC003480) derived from GenBank at the nucleic acid level, respectively. It was also indicated that 63.7 to $64.3 \%, 57.4$ to $58.6 \%$, and 57.8 to $58.8 \%$ identical to the RNA1 to 3 segments of PNRSV (accession nos. NC004362, NC004363, and NC004364), respectively. The dendrogram analysis of ApNMV with ApMV and PNRSV with all available nucleotide sequences in GenBank were constructed based on MP and CP genes (Supplementary Fig. S2). ApNMV was distantly related to ApMV and PNRSV at the nucleotide and amino acid sequences level. While ApMV-PV32 (accession no. U03857) was found to be clustered with PNRSV rather than with ApMV, and it might be regarded as a strain of PNRSV, as previously reported (Guo et al. 1995).

Occurrence and distribution of ApNMV in China. In a preliminary report (Noda et al. 2017), ApNMV was described as detected in 228 out of 276 apple samples displaying mosaic symptoms. In the present study, a more detailed analysis of the virus status of the symptomatic apple samples from that survey was performed (Table 1). The high-quality RNA was extracted using RNAprep pure plant kit, and ApNMV was additionally detected in 26 out of 28 negative samples tested in the preliminary study and in 14 out of 15 symptomatic samples additionally collected in this study, by RT-PCR and nested RTPCR. Together, a total of 268 symptomatic samples (92.1\%) tested positive for ApNMV (Table 1). The distribution of apple mosaic samples collected from six provinces and regions that tested positive for ApNMV is shown in Table 1. These sampled provinces and regions contribute to about $68.7 \%$ of the total apple production in China (Shaanxi 25.1\%, Shandong 22.3\%, Shanxi 9.8\%, Gansu $8.2 \%$, Xinjiang $3.1 \%$, Beijing $0.2 \%$, official data from National Bureau of Statistics of the People's Republic of China). No samples obtained from trees showing mosaic symptoms were positive for ApMV by RT-PCR using three primer sets (Supplementary Table S2). In previous studies, ApMV was detected using the primer set ILAR1/2, designed by Candresse et al. (1998), from apple mosaic samples in China (Hu et al. 2015; Ji et al. 2013). However, multiple sequence alignment analysis and PCR results showed that the primer set ILAR1/2 could also be used for the detection of ApNMV (Supplementary Fig. S3). Therefore, ApNMV may have been identified as

Table 3. Genome organization of Apple necrotic mosaic virus (ApNMV) isolated from apple trees showing mosaic symptom in China

\begin{tabular}{|c|c|c|c|c|c|c|c|c|c|c|c|c|c|c|}
\hline \multirow[b]{2}{*}{ Isolate } & \multicolumn{4}{|c|}{ RNA1 } & \multicolumn{4}{|c|}{ RNA2 } & \multicolumn{6}{|c|}{ RNA3 } \\
\hline & Length $^{a}$ & 5'UTR & MET/HEL $^{b}$ & 3'UTR $^{\prime}$ & Length $^{\mathbf{a}}$ & 5'UTR & POL $^{\mathrm{c}}$ & $\mathbf{3}^{\prime}$ UTR & Length $^{\mathbf{a}}$ & 5'UTR & MPd $^{\mathbf{d}}$ & $\mathbf{I R}^{\mathbf{e}}$ & $\mathbf{C P}^{\mathbf{f}}$ & $3^{\prime}$ UTR \\
\hline AM27 & 3,380 & 51 & 3,171 & 158 & 2,786 & 58 & 2,568 & 160 & 1,954 & 188 & 843 & 103 & 660 & 160 \\
\hline AM36 & 3,380 & 51 & 3,171 & 158 & 2,786 & 58 & 2,568 & 160 & 1,955 & 189 & 843 & 103 & 660 & 160 \\
\hline AM72 & 3,378 & 50 & 3,171 & 157 & 2,782 & 54 & 2,568 & 160 & 1,909 & 147 & 843 & 99 & 660 & 160 \\
\hline AM75 & 3,378 & 50 & 3,171 & 157 & 2,778 & 52 & 2,595 & 131 & 1,916 & 150 & 843 & 102 & 660 & 161 \\
\hline AM95 & 3,379 & 51 & 3,171 & 157 & 2,785 & 57 & 2,568 & 160 & 1,915 & 149 & 843 & 103 & 660 & 160 \\
\hline AM125 & 3,378 & 50 & 3,171 & 157 & 2,781 & 52 & 2,604 & 125 & 1,915 & 150 & 846 & 98 & 660 & 161 \\
\hline
\end{tabular}

${ }^{a}$ Total length in nucleotides.

b Methyltransferase/NTP-binding helicase.

${ }^{\mathrm{c}}$ RNA polymerase.

${ }^{\mathrm{d}}$ Movement protein.

e Intergenic region.

${ }^{\mathrm{f}}$ Coat protein.

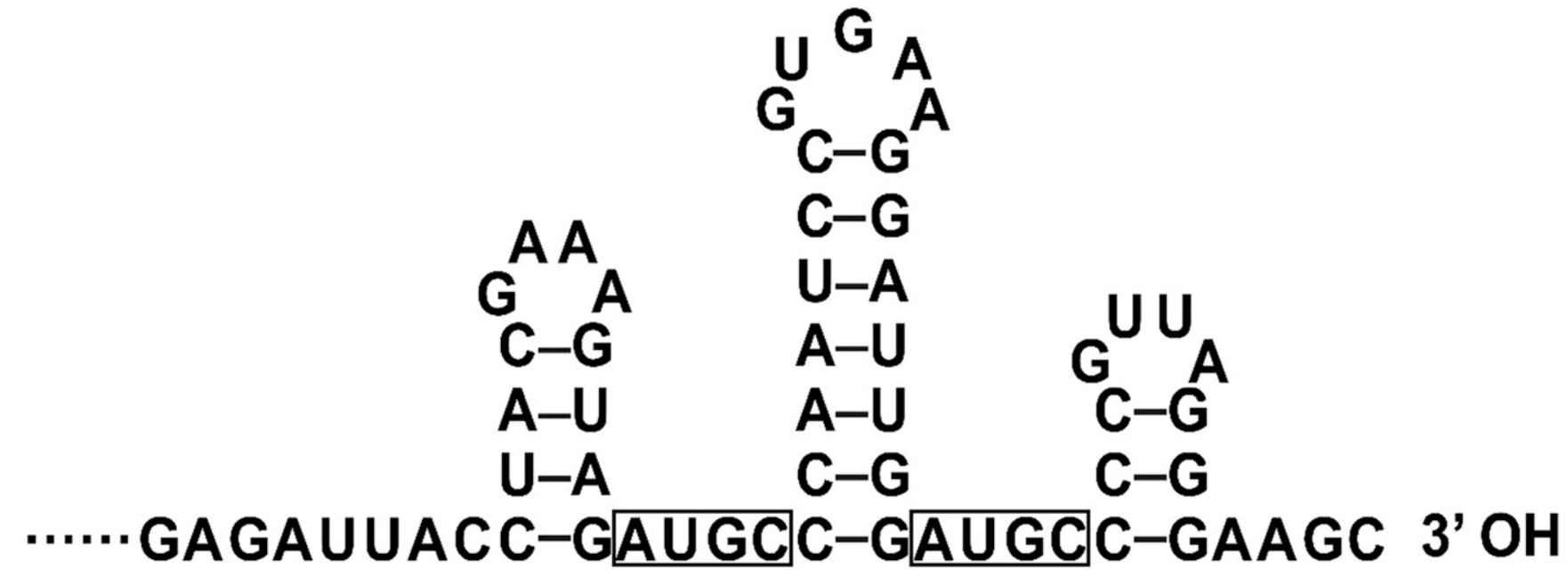

Fig. 2. Putative secondary structure (above) and nucleotide alignment (below) of the $3^{\prime}$-UTR of Apple necrotic mosaic virus (ApNMV). The stem-loop sequences and motifs were indicated with shadow and black box, respectively. 

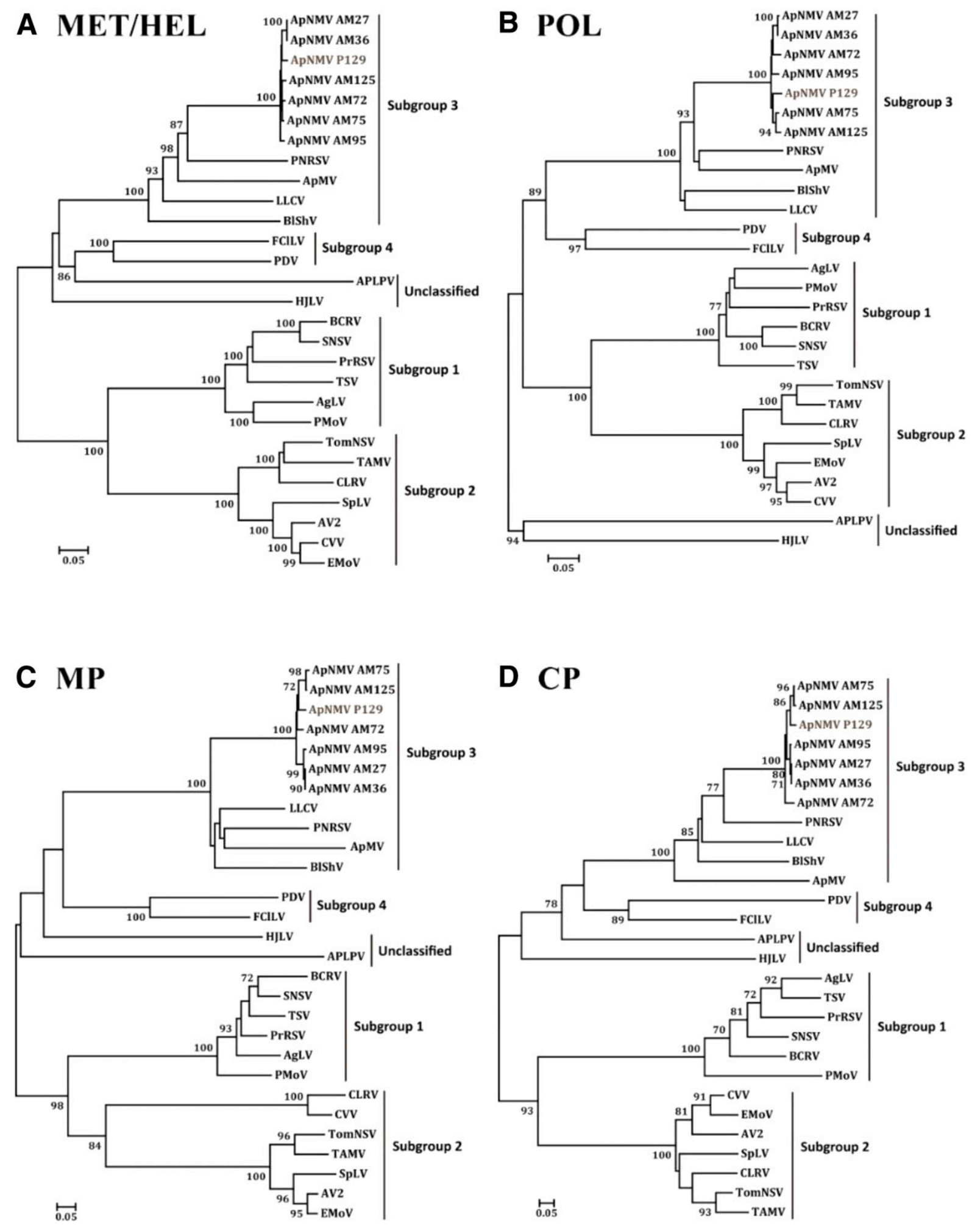

Fig. 3. Phylogenetic relationship of the Chinese Apple necrotic mosaic virus (ApNMV) isolates (AM27, 36, 72, 75, 95, and 125) with members of the genus llarvirus. The tree was constructed using the neighbor-joining method with 1,000 bootstrap replications based on the amino acids of $\mathbf{A}$, methyltransferase/NTP-binding helicase (MET/HEL); $\mathbf{B}$, RNA polymerase (POL); C, movement protein (MP); and D, coat protein (CP). Only bootstrap values $\geq 70 \%$ were shown. Subgroup 1: Ageratum latent virus (AgLV, NC022127, NC022128, and NC022129), Blackberry chlorotic ringspot virus (BCRV, NC011553, NC011554, and NC011555), Parietaria mottle virus (PMoV, NC005848, NC005849, and NC005854), Privet ringsport virus (PrRSV, NC027928, NC027929, and NC027930), Strawberry necrotic shock virus (SNSV, NC008708, NC008707, and NC008706), Tobacco streak virus (TSV, NC003844, NC003842, and NC003845). Subgroup 2: Asparagus virus 2 (AV2, NC011808, NC011809, and NC011807), Citrus leaf rugose virus (CLRV, NC003548, NC003547, and NC003546), Citrus variegation virus (CVV, NC009537, NC009538, and NC009536), Elm mottle virus (EMoV, NC003569, NC003568, and NC003570), Spinach latent virus (SpLV, NC003808, NC003809, and NC003810), Tomato necrotic streak virus (TomNSV, KT779204, KT779205, and KT779206), Tulare apple mosaic virus (TAMV, NC003833, NC003834, and NC003835). Subgroup 3: Apple mosaic virus (ApMV, NC003464, NC003465, and NC003480), Blueberry shock virus (BIShV, NC022250, NC022251, and NC022252), Lilac leaf chlorosis virus (LLCV, NC025477, NC025478, and NC025481), Prunus necrotic ringspot virus (PNRSV, NC004362, NC004363, and NC004364). Subgroup 4: Fragaria chiloensis latent virus (FCILV, NC006566, NC006567, and NC006568), Prune dwarf virus (PDV, NC008039, NC008037, and NC008038). No subgroup determined: American plum line pattern virus (APLPV, NC003451, NC003452, and NC003453), Humulus japonicus latent virus (HJLV, NC006064, NC006065, and NC006066). Japanese ApNMV isolate (P129, LC108993, LC108994, and LC108995) are shown in gray. 
ApMV by mistake (Hu et al. 2015; Ji et al. 2013). We also tested 75 apple mosaic samples by RT-PCR for the presence of other viruses and a viroid widely distributed in China (ASPV, ASGV, ACLSV, and ASSVd). Out of the 75 tested samples, all were infected by ApNMV (100\%), 49 were infected by ASPV (65.3\%), 74 were infected by ASGV (98.7\%), 52 were infected by ACLSV (69.3\%), and seven were infected by ASSVd (9.3\%). The mixed infection of at least three viruses/viroid was very common (84\%). Only one sample showing mosaic disease was separately infected by ApNMV. On the whole, this indicated that ApNMV occurred widely in most apple-producing areas in China, and it may be the main culprit of apple mosaic disease in China rather than ApMV.

\section{Discussion}

Apple mosaic disease is widely distributed all over the world, leading to major adverse effects to the yield and quality of apple fruits ( $\mathrm{Li}$ et al. 2002; Wood et al. 1975). ApMV has been reported to cause mosaic disease on apple trees (Chamberlain et al. 1964; Fulton 1972; Grimová et al. 2016; Petrzik and Lenz 2011), but the causal agent of apple mosaic disease in China was still unclear. In previous reports, ApMV, which was regarded as the causal agent of apple mosaic disease, was detected from apple trees in China by RT-PCR using the polyvalent primers (Candresse et al. 1998; Hu et al. 2015; Ji et al. 2013). These primers are not only suitable for the detection of ApMV, but would also be for ApNMV based on the sequence and alignment analysis. It is possible that misidentification of ApMV as the cause of the apple mosaic disease would be explained as a result of misannealing of the ApMV specific primers. We speculate that ApNMV was erroneously reported as ApMV in China. From the extensive survey in China, nearly 300 apple samples with mosaic symptoms were not positive for ApMV using the specific-primer sets. Interestingly, the mosaic symptoms of ApMV and ApNMV are indistinguishable on apple leaves, and it is possible that different pathogens may cause the same or similar symptoms when the identical key gene regulatory networks were induced. We also found that ApNMV and the three latent viruses (ASPV, ASGV, and ACLSV), which were usually symptomless in most commercial apple cultivars (Jelkmann and Paunovic 2011; Massart et al. 2011; Nemeth 1986; Yaegashi et al. 2011), occurred frequently in mixed infections in perennial woody apple plants. Our study suggested that ApNMV was highly associated with apple mosaic disease rather than ApMV in China, because as yet we could not detect ApMV in apple samples displaying mosaic disease. Similarly, in the recent brief review for ApMV distribution and incidence, ApMV was not reported from apple trees in China (Grimová et al. 2016).

ApNMV showed a strong association with apple mosaic diseases in China. In addition, only a few samples showed necrotic symptoms among all tested samples positive for ApNMV. At this stage, it is difficult to generalize that the necrotic symptoms are caused by ApNMV. This can be proven if similar symptoms can be observed in following pathogenicity studies on the apple seedlings inoculated with infectious clones. In our previous study, the incidence of ApNMV in apple trees with mosaic symptoms was $82.6 \%$ (228 out of 276 samples) (Noda et al. 2017). In the present study, however, we found that relatively higher incidence rates $(92.1 \%, 268$ out of 291 samples) of ApNMV in tested samples. Total RNA extraction using RNAprep pure plant kit increases the number of ApNMV-positive samples showing mosaic disease. The kits may reduce or eliminate inhibitory substances for PCR amplification, such as polysaccharides and polyphenolics. Thus, high quality RNA preparations are needed to improve the detection of ApNMV and avoid false-negative results.

In this study, the complete nucleotide sequences of the six ApNMV isolates from different cultivars and geographical areas in China were determined and analyzed. The genome of ApNMV consisted of three RNA molecules ranging in size from 3,378 to $3,380 \mathrm{nt}, 2,778$ to $2,786 \mathrm{nt}$, and 1,909 to $1,955 \mathrm{nt}$, respectively. Interestingly, the RNA3 segment of ApNMV showed high variability at the 5' UTR. About 40 nucleotides were inserted/deleted in the $5^{\prime}$ UTR while MP and CP genes were relatively stable over the nucleotide length. At first, we attributed it to different cultivars from which the isolates were obtained, but this may not be the reason after we obtained four additional complete nucleotide sequences of RNA3 (1,909 to 1,920 nt in length, data not shown). In addition, we found that the IRs of the complete nucleotide sequences of six Chinese ApNMV isolates were shorter than that of the Japanese isolate, since $46 \mathrm{nt}$ repetitive sequences were found out at position 1,068 to 1,113 and position 1,114 to 1,159 in the RNA3 segment of the Japanese isolate P129. Multiple sequence alignment revealed that $3^{\prime}$ UTRs were highly conserved and contained a putative stem-loop structure that may play a crucial role in virus replication.

ApNMV belongs to subgroup 3 of Ilarvirus with typical genomic characteristics, as shown by phylogenetic analyses of the four genes at the nucleotide and amino acid levels. Compared with other ilarviruses, MP showed the highest similarity to LLCV at the amino acid levels, whereas it showed the highest nucleotide identity to PNRSV (60.6 to 62\%). Considering the close relationship of the viruses infecting apple trees, MP and CP genes of the accepted PNRSV and ApMV available until now in GenBank were used to construct the phylogenetic trees. All complete nucleotide sequences obtained in this study supported grouping of ApNMV in subgroup 3 of the genus Ilarvirus. It showed that ApNMV had a closer phylogenetic relationship to PNRSV than to ApMV but distinguished from them.

In summary, we concluded that the full genomes of the six Chinese ApNMV isolates showed sequence diversities, especially in RNA3. This is the first report of the complete nucleotide sequences of ApNMV in China. The complete nucleotide sequences of ApNMV obtained in this study would be helpful for further study on virus taxonomic position of ApNMV. ApNMV was distinct from ApMV and PNRSV. So far, the full-length cDNA clones of ApNMV have been constructed and will be inoculated to apple seedlings in the near future. Furthermore, host range of ApNMV in woody tree species needs to be determined. It is also essential to study the pathogenicity of various strains of ApNMV in apple trees.

\section{Acknowledgments}

We want to acknowledge Prof. Robert Owens (U. S. Department of Agriculture, Beltsville, MD, United States) and Nuredin Habili (from The University of Adelaide, Australia) for their valuable comments and suggestions, especially to improve the language of our manuscript.

\section{Literature Cited}

Bradford, F. C., and Joley, L. 1933. Infectious variegation in the apple. J. Agric. Res. 46:901-908.

Candresse, T., Kofalvi, S. A., Lanneau, M., and Dunez, J. 1998. A PCR-ELISA procedure for the simultaneous detection and identification of prunus necrotic ringspot (PNRSV) and apple mosaic (ApMV) ilarviruses. Acta Hortic. 472:219-226.

Chamberlain, E. E., Atkinson, J. D., and Hunter, J. A. 1964. Cross-protection between strains of Apple mosaic virus. N. Z. J. Agric. Res. 7:480-490.

Fukushi, T., and Tahama, Y. 1960. On apple mosaic. Mem FacAgric Hokkaido Univ. 3:116-123.

Fulton, R. W. 1972. Apple mosaic virus. CMI/AAB Description Plant Viruses No. 83. Association of Applied Biologists, Wellesbourne, U.K.

Grimová, L., Winkowska, L., Konrady, M., and Ryšánek, P. 2016. Apple mosaic virus. Phytopathol. Mediterr. 55:1-19.

Guo, D., Maiss, E., Adam, G., and Casper, R. 1995. Prunus necrotic ringspot ilarvirus: nucleotide sequence of RNA3 and the relationship to other ilarviruses based on coat protein comparison. J. Gen. Virol. 76:1073-1079.

Hammond, R. W., and Crosslin, J. M. 1995. The complete nucleotide sequence of RNA 3 of a peach isolate of prunus necrotic ringspot virus. Virology 208: 349-353.

Herranz, M. C., and Pallas, V. 2004. RNA-binding properties and mapping of the RNA-binding domain from the movement protein of prunus necrotic ringspot virus. J. Gen. Virol. 85:761-768.

Hu, G. J., Zhang, Z. P., Dong, Y. F., Fan, X. D., Ren, F., and Zhu, H. J. 2015 Efficiency of virus elimination from potted apple plants by thermotherapy coupled with shoot-tip grafting. Australas. Plant Pathol. 44:167-173.

James, D., Varga, A., Leippi, L., Godkin, S., and Masters, C. 2010. Sequence analysis of RNA 2 and RNA 3 of Lilac leaf chlorosis virus: a putative new member of the genus Ilarvirus. Arch. Virol. 155:993-998.

Jelkmann, W., and Paunovic, S. 2011. Apple stem pitting virus. Pages 35-40 in: Virus and Virus-Like Diseases of Pome and Stone Fruits. A. Hadidi, M. Barba, T. Candresse, and W. Jelkmann, eds. APS Press, St. Paul, MN.

Ji, Z., Zhao, X., Duan, H., Hu, T., Wang, S., Wang, Y., and Cao, K. 2013 Multiplex RT-PCR detection and distribution of four apple viruses in China Acta Virol. 57:435-441. 
Li, D. H., Zhao, H. Y., Hu, Z. Q., Hu, X. S., and Zhang, Y. H. 2002. Studies on the damage, loss of production and control to apple mosaic disease. J. NW Sci. Tech. Univ. Agric. For. (Nat. Sci. Ed.) 30:77-80.

Liang, P. B., Zhang, Z. X., Liu, F., Lu, M. G., Li, S. F., and Wang, H. Q. 2016. Identification of pathogens associated with apple mosaic symptom. J. Fruit Sci. 33:257-267.

Lin, L. M., Li, R. H., Mock, R., and Kinard, G. 2011. Development of a polyprobe to detect six viroids of pome and stone fruit trees. J. Virol. Methods 171:91-97.

Massart, S., Jijakli, M. H., and Kummert, J. 2011. Apple stem grooving virus. Pages 29-33 in: Virus and Virus-Like Diseases of Pome and Stone Fruits. A. Hadidi, M. Barba, T. Candresse, and W. Jelkmann, eds. APS Press, St. Paul, $\mathrm{MN}$.

Nemeth, M. 1986. Page 841 in: Virus, Mycoplasma and Ricketsia Diseases of Fruit Trees. Martinus Nijhoff Publishers, Boston.

Noda, H., Yamagishi, N., Yaegashi, H., Xing, F., Xie, J. P., Li, S. F., Zhou, T., Ito, T., and Yoshikawa, N. 2017. Apple necrotic mosaic virus, a novel ilarvirus from mosaic-diseased apple trees in Japan and China. J. Gen. Plant Pathol. 83:83-90.

Pallas, V., Aparicio, F., Herranz, M. C., Amari, K., Sanchez-Pina, M. A., Myrta, A., and Sanchez-Navarro, J. A. 2012. Ilarviruses of Prunus spp.: a continued concern for fruit trees. Phytopathology 102:1108-1120.
Pallas, V., Aparicio, F., Herranz, M. C., Sanchez-Navarro, J. A., and Scott, S. W. 2013. The molecular biology of ilarviruses. Adv. Virus Res. 87:139-181.

Petrzik, K., and Lenz, O. 2011. Apple mosaic virus in pome fruits. Pages 25-28 in: Virus and Virus-Like Diseases of Pome and Stone Fruits. A. Hadidi, M. Barba, T. Candresse, and W. Jelkmann, eds. APS Press, St. Paul, MN.

Saitou, N., and Nei, M. 1987. The neighbor-joining method: a new method for reconstructing phylogenetic trees. Mol. Biol. Evol. 4:406-425.

Sanchez-Navarro, J. A., and Pallas, V. 1994. Nucleotide sequence of apple mosaic ilarvirus RNA 4. J. Gen. Virol. 75:1441-1445.

Tamura, K., Stecher, G., Peterson, D., Filipski, A., and Kumar, S. 2013. MEGA6: molecular evolutionary genetics analysis version 6.0. Mol. Biol. Evol. 30: 2725-2729.

Wood, G. A., Chamberlain, E. E., Atkinson, J. D., and Hunter, J. A. 1975. Field studies with Apple mosaic virus. N. Z. J. Agr. Res. 18:399-404.

Yaegashi, H., Yoshikawa, N., and Candresse, T. 2011. Apple chlorotic leaf spot virus in pome fruits. Pages 17-21 in: Virus and Virus-Like Diseases of Pome and Stone Fruits. A. Hadidi, M. Barba, T. Candresse, and W. Jelkmann, eds. APS Press, St. Paul, MN.

Zhang, Z. X., Peng, S., Jiang, D. M., Pan, S., Wang, H. Q., and Li, S. F. 2012. Development of a polyprobe for the simultaneous detection of four grapevine viroids in grapevine plants. Eur. J. Plant Pathol. 132:9-16. 